\title{
Antibiotic resistance assessment of Acinetobacter baumannii isolates from Tehran hospitals due to the presence of efflux pumps encoding genes (adeA and ades genes) by molecular method
}

\author{
Batool Basatian-Tashkan ${ }^{1}$, Mohammad Niakan ${ }^{1 *}$, Mansoor Khaledi ${ }^{1}$, Hamed Afkhami ${ }^{1}$, Fatemeh Sameni ${ }^{1}$,
} Shahriar Bakhti ${ }^{1}$ and Reza Mirnejad ${ }^{2}$

\begin{abstract}
Objective: Acinetobacter baumannii (A. baumannii) has caused many problems in nosocomial infections. Efflux pumps are considered as one of the most important mechanisms of resistance in this bacterium and have the ability to excrete toxic substances such as antibiotics out of the cell.

Results: In this study, 60 isolates of A. baumannii were collected from patients in several hospitals in Tehran, Iran. After diagnosis using standard biochemical methods, the pattern of antibiotic susceptibility was determined using the disk diffusion method according to CLSI guidelines. The adeA and adeS genes were identified by PCR method. The highest resistance to Piperacillin and the lowest resistance to Gentamicin were observed (100\% compared to 48.4\%). $6.6 \%$ of the isolates had only adeA gene and adeS gene was observed in $8.4 \%$ of isolates and both genes were detected in $73.4 \%$ of the samples. Despite the high resistance of $t$ A. baumannii o antibiotics and due to the high frequency of genes of adeA and adeS efflux pumps in A. baumannii isolates, it can be concluded that these efflux pumps may play an important role in resistance of this bacterium. By determining the pattern of antibiotic the resistance before treatment, the resistance of this pathogen can be prevented in societies.
\end{abstract}

Keywords: Acinetobacter baumannii, Efflux pump, adeA gene, adeS gene, Antibiotic resistance

\section{Introduction}

Nosocomial infections are known as one of the crucial problems for public health [1]. Most of these infections are caused by Gram-negative bacilli [2]. One of the most important causes of nosocomial infections is A. baumannii that is Gram-negative Coccobacilli and aerobic and prefer humid environments for a living $[3,4]$. The

\footnotetext{
*Correspondence: niakan@shahed.ac.ir

${ }^{1}$ Department of Microbiology, Faculty of Medicine, Shahed University, Tehran, Iran

Full list of author information is available at the end of the article
}

most important pathogen of this genus is, A. baumannii which can cause a wide range of diseases and nosocomial infections such as pneumonia, septicemia, urinary tract infections, skin and wound infections, endocarditis, and meningitis $[5,6]$. Infection with this bacterium is increasing in people with immunodeficiency or cancer [7]. One of the main problems caused by this bacterium is the development of high antibiotic resistance [8]. One of the mechanisms that this bacterium uses to resist various antibiotics is the use of efflux pumps. By using efflux pumps, the A. baumannii can direct antibiotics outwards and prevent antibiotics from affecting the bacteria [9].

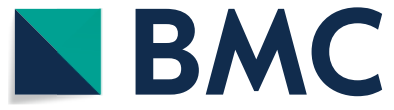

(c) The Author(s) 2020. This article is licensed under a Creative Commons Attribution 4.0 International License, which permits use, sharing, adaptation, distribution and reproduction in any medium or format, as long as you give appropriate credit to the original author(s) and the source, provide a link to the Creative Commons licence, and indicate if changes were made. The images or other third party material in this article are included in the article's Creative Commons licence, unless indicated otherwise in a credit line to the material. If material is not included in the article's Creative Commons licence and your intended use is not permitted by statutory regulation or exceeds the permitted use, you will need to obtain permission directly from the copyright holder. To view a copy of this licence, visit http://creativeco mmons.org/licenses/by/4.0/. The Creative Commons Public Domain Dedication waiver (http://creativecommons.org/publicdomain/ zero/1.0/) applies to the data made available in this article, unless otherwise stated in a credit line to the data. 
Due to the high prevalence of efflux pump genes especially AdeA and AdeB that are the major discovered efflux pumps in $A$. baumannii, this may play a significant role in the antibiotic resistance of $A$. baumannii isolates. Identifying the antibiotic susceptibility pattern is essential to prevent the prevalence of antibiotic resistance. For this purpose, this study was performed by determining the antibiotic susceptibility in clinical trials of $A$. baumannii collected from several hospitals in Tehran.

\section{Main text}

\section{Materials and methods}

\section{Patients and sampling}

In this study, $60 \mathrm{~A}$. baumannii isolates were collected from 100 different samples including chips, blood, urine, wound culture, respiratory secretions, catheters, spinal fluid, and pleurisy during the 9 months from August 2018 to May 2019 from different departments of Milad Hospital, Baqiyatallah and the Rasool-Akram hospital of Tehran was identified.

1. Isolation and identification of $A$. baumannii strains Prevalent biochemical tests and methods have been used to identify and confirm $A$. baumannii strains [10].

The specimens were inoculated on blood agar (Merck) and MacConkey agar (Merck) medium and incubated for $24 \mathrm{~h}$ at $37^{\circ} \mathrm{C}$. Conventional biochemical methods such as oxidase, citrate, urea urease, malonate consumption, oxidation and fermentation of sugars, motility, and indole production were done to identify A. baumannii [11].

2. Confirmation of Acinetobacter baumannii by PCR To confirm the Acinetobacter baumannii, the gene bla OXA-51-like was examined by PCR similar to the method performed by Jia et al. [12]. Because it has been made clear that, there is OXA-51-like gene in Acinetobacter baumannii isolates instinctively [13].

\section{Antimicrobial susceptibility test}

This test was performed using the Kirby Bauer method using 9 disks including Imipenem $(10 \mu \mathrm{g})$, meropenem $(10 \mu \mathrm{g})$, Gentamicin, Piperacillin, Ampicillin-Sulbactam, Ceftazidime, Amikacin, Tetracycline, and Ciprofloxacin. The 2019 CLSI was interpreted [14]. The Acinetobacter baumanii ATCC19606 was used as quality control for this test.

\section{DNA extraction}

DNA extracting was performed by DNA extraction kit (Bioneer Company Korea, Cat. No. K-3032-2-) was used.

\section{Identification of adeA and adeS gene via PCR}

PCR was performed to screen for adeA and adeS genes. The primer sequences used are shown in Table 1. The PCR program For amplification was: an initial denaturing step, 5 min at $94{ }^{\circ} \mathrm{C}, 30$ cycles of the $30 \mathrm{~s}$ at $94{ }^{\circ} \mathrm{C}$, primer connection for adeA at $55.5^{\circ} \mathrm{C}$ and For adeS at $54.5^{\circ} \mathrm{C}$ for the $30 \mathrm{~s}, 90 \mathrm{~s}$ at $72{ }^{\circ} \mathrm{C}$, and $5 \mathrm{~min}$ as a final extension at $72{ }^{\circ} \mathrm{C}$ were performed. The PCR products were analyzed through a $2 \%$ agarose gel containing Syber safe and were detected using a gel documentary device.

The primers designed in this study are as follows: VIM-1 5'-TGGTTGTATACGTCCCGTCA TGTGTG CTGGAGCAAGTCTA-3', IMP-15'-TAACGGGTGGGG CGTTGTTCCT CGCCCGTGCTGTCGCTATGAAA- ${ }^{\prime}$ and The third primer for OXA-51 was described previously [11].

Primers for adeA and adeS genes:

adeA Forward $5^{\prime}$-TTGATCGTGCTTCTATTCCTC AAG- $3^{\prime}$

adeA Reverse 5'-GGCTCGCCACTGATATTACGTT$3^{\prime}[15]$

adeS Forward 5' - TGCCGCCAAATTCTTTATTC-3'

adeS Reverse 5'- TTAGTCACGGCGACCTCTCT-3' [16].

\section{Statistical analysis}

Questionnaire information and the results of phenotypic and genotypic experiments were analyzed using SPSS software, version 23, by the Chai test and Fisher's exact test.

\section{Results}

In this study, 60 bacterial isolates were collected as Acinetobacter baumanii from three hospitals in Tehran. The frequency distribution of Acinetobacter baumanii Isolates according to the sample is shown in Table 1.

Table 1 Frequency distribution of Acinetobacter baumanii isolated from patients according to the type of clinical sample and hospital

\begin{tabular}{llllc}
\hline Sample type & Baqiyatallah & $\begin{array}{l}\text { Rasool- } \\
\text { Akram }\end{array}$ & Milad & N (\%) \\
\hline Tracheal & 2 & 1 & 1 & $4(6.6)$ \\
Wound & 6 & 3 & 4 & $13(21.8)$ \\
Urine & 3 & 2 & 2 & $7(11.8)$ \\
Blood & 6 & 5 & 5 & $16(26.6)$ \\
Catheter & 1 & 1 & 2 & $4(6.6)$ \\
& 7 & 3 & 6 & $16(26.6)$ \\
\hline
\end{tabular}




\section{Antibiogram results by agar disc diffusion method}

Out of $60 \mathrm{~A}$. baumannii isolates, all of them were resistant to Piperacillin, and this rate was as follows in other antibiotics: Ceftazidime 98.4\%, Amikacin 96.6\%, Tetracycline 91.6\%, Ampicillin-Sulbactam 65\%, Meropenem 63.4\%, Ciprofloxacin 61.6\%, Imipenem 50\%, and Gentamicin $48.4 \%$, which had the considerable effect and Piperacillin and Ceftazidime showed the most resistance levels (Table 2).

\section{Results of genotypic tests}

The presence of the OXA-51 gene in the Acinetobacter baumannii was investigated. All samples in the PCR method had this gene. The frequency distribution of the ade $A$ and adeS genes in the PCR method was also examined. The frequency of adeA and adeS genes in the samples were $6.6 \%$ and $8.4 \%$, respectively calculated by PCR method. In $44(73.4 \%)$ of the samples, these two genes were present together and in 7 (11.6\%) of the samples, none of the adeA and adeS genes were present.

\section{Results of the relationship between antibiotic resistance and efflux pump genes}

According to the results, it can be concluded that the expression of adeA and adeS genes are related to the Tetracycline, Ciprofloxacin, Gentamicin, and Amikacin resistance (Table 3).

Studies show that in addition to efflux ade $A B C$ pumps, other efflux pumps also have a leading role to play in developing resistance to these antibiotics, and the simultaneous presence of these efflux pumps increases resistance. Although other factors contribute to this antibiotic resistance, efflux pumps play the most crucial role in creating resistance to these antibiotics [17].

Table 2 Antibiotic resistance pattern of Acinetobacter baumanii isolates

\begin{tabular}{llll}
\hline Antibiotic & Resistant N (\%) & $\begin{array}{l}\text { Intermediate } \\
\mathbf{N}(\%)\end{array}$ & Susceptible N (\%) \\
\hline Piperacillin & $60(100)$ & $0(0)$ & $0(0)$ \\
Ceftazidime & $59(98.4)$ & $1(1.6)$ & $0(0)$ \\
Amikacin & $58(96.6)$ & $0(0)$ & $2(3.4)$ \\
Tetracycline & $55(91.6)$ & $2(3.4)$ & $3(5)$ \\
Ampicillin- & $39(65.0)$ & $2(3.4)$ & $19(31.6)$ \\
Sulbactame & & $1(1.6)$ & $21(35)$ \\
Meropenem & $38(63.4)$ & $3(5.0)$ & $20(33.4)$ \\
Ciprofloxacin & $37(61.6)$ & $0(0)$ & $30(50.0)$ \\
Imipenem & $30(50.0)$ & $0(0)$ & $31(21.6)$ \\
Gentamicin & $29(48.4)$ & & \\
\hline
\end{tabular}

Table 3 Antibiotic resistance in acinetobacter bacterial isolates

\begin{tabular}{llll}
\hline Antibiotic & Resistant N (\%) & $\begin{array}{l}\text { Resistant. ade } \\
\text { A gene (\%) }\end{array}$ & $\begin{array}{l}\text { Resistant. } \\
\text { ade S gene } \\
\text { (\%) }\end{array}$ \\
\hline Amikacin & $58(96.6)$ & 48 & 49 \\
Tetracycline & $55(91.6)$ & 36 & 36 \\
Ciprofloxacin & $37(61.6)$ & 29 & 29 \\
Gentamicin & $29(48.4)$ & 48 & 49 \\
\hline
\end{tabular}

\section{Discussion}

Nosocomial infections have become one of the main problems in treatment. The most common cause of nosocomial infections is Gram-negative bacteria. Acinetobacter baumannii is an opportunistic hospital pathogen that causes a wide range of nosocomial infections. Due to the indiscriminate use of broad-spectrum antibiotics by people, we are witnessing high antibiotic resistance caused by this bacterium. The high antibiotic resistance of this bacterium is associated with the proliferation of multiple antibiotic resistance genes. Various studies have shown that Acinetobacter baumannii is resistant to most Betalactam antibiotics and Quinolones, and its resistance to Aminoglycosides is increasing. In this study, the most effective antibiotics used against Acinetobacter baumannii were Gentamicin and Imipenem, which had $48.4 \%$ and $50 \%$ resistance, respectively, while the highest resistance was to Piperacillin (100\%), Ceftazidime (98.4\%), and Amikacin (96.6\%) and Tetracycline (91.6\%).

In the present study, the resistance of Acinetobacter baumannii to at least one antibiotic was seen in three groups or more in all isolates. Therefore, the frequency of multi-drug resistance in Acinetobacter baumannii isolates was $100 \%$, but the frequency of Acinetobacter baumannii with multiple resistance in other studies reported was reported to be 50 to $100 \%$ variable [12]. High MDR levels in Acinetobacter baumannii studied in Farsiani et al. Studies in Iran and Rynga et al. In India were reported to be $97 \%$ and $85 \%$, respectively $[18,19]$, which could be due to abuse of antibiotics.

In the Noori et al. study, All isolates had 100\% resistance to Ceftazidime, Ciprofloxacin, and Piperacillin, and the frequency of the adeS gene was 91\% which in comparison to Ciprofloxacin adeS resistance was different [20]. According to the results of the present study, we saw a decrease in antibiotic resistance to ceftazidime and ciprofloxacin and the same resistance to piperacillin was reported.

Research by Rahbarnia et al. Found that the resistance to Ciprofloxacin was 95\%, Imipenem $82 \%$, and Gentamicin 35\%. Also, the prevalence of MDR and XDR 
in the studied strains was $76 \%$ and $30 \%$, respectively, which compared to the present study, higher resistance to Ciprofloxacin and Imipenem has been reported [21]. This reason for the increase in antibiotic resistance may be related to different geographical areas in Iran.

In Abdar et al. study, the resistance to Meropenem and Ceftazidime was reported to be $71 \%$ and $93 \%$, respectively, which is very similar to the present study [22].

According to Fallah et al., The antibiotic resistance of Acinetobacter baumanii isolates is as follows: $95.4 \%$ to Ceftazidime, $91.7 \%$ to Meropenem, and $92.6 \%$ to Ciprofloxacin, which is reported to have a higher resistance to Meropenem and Ciprofloxacin than the present study [23]. The reason for the decrease in antibiotic resistance in recent years in Iran can be due to changes in antibiotic treatment policies and the use of antibiograms before prescribing antibiotics.

According to a report by Al-Agamy et al., $100 \%$ of Acinetobacter baumanii isolates were $85 \%$ resistant to Ciprofloxacin and $70 \%$ to Imipenem [24]. Compared to the present study, we saw a decrease in resistance to both antibiotics.

According to the study of Angoti et al., The resistance to Ciprofloxacin is $99 \%$, Ceftazidime, Meropenem, and Imipenem 98\%, Gentamicin 77\%, Amikacin 48\% which is different from the present study and also the prevalence of adeA gene in 61 samples $88.5 \%$ was reported [25]. Based on the results of a recent study, we have seen a decrease in antibiotic resistance in recent years in the treatment of patients.

In a study by Boral et al. [26], antibiotic resistance for Ciprofloxacin, Imipenem, Ampicillin/sulbactam, Ceftazidime, and Amikacin was observed to be $100 \%, 99.4 \%$, $99.4 \%, 99.4 \%$, and $91.8 \%$, respectively. That is an increase in reported antibiotic resistance compared to the present study.

In a study by Ranjbar et al., The antibiotic resistance to Ceftazidime, Ciprofloxacin, Piperacillin, Gentamicin, Amikacin, and Ampicillin/Sulbactam was reported to be $97.5 \%, 96.3 \%, 95.1 \%, 92.1 \%, 87.2 \%$, and $76.1 \%$, respectively [27]. One of the reasons for the difference in antibiotic resistance compared to the recent study is the diverse geographical areas for sampling patients.

In a study by Jia et al. China, resistance to Piperacillin and Ceftazidime was reported $92.2 \%$ that was similar to this study [12].

The frequency of Tetracycline resistance in the studied isolates was $91.6 \%$. The results of studies conducted in Iran were similar to the results of studies of Sarhaddi et al. [28] that were $96.3 \%$ and compared to the study of Nemec et al. Antibiotics were 62\% higher [29].
One of the resistance mechanisms in the Acinetobacter baumanii is the presence of efflux pumps. These pumps cause the leakage of antibiotics and a wide range of substances out of the bacteria, creating multidrug resistance. Three systems, AdeFGH: RND, AdeIJK, and $A d e A B C$, have been identified in the Acinetobacter baumanii, among which $A d e A B C$ is most involved in the MDR Acinetobacter baumanii [30]. Although high levels of resistance do not occur only as a result of multi-drug efflux pumps, the expression of their genes among isolates with high antibiotic resistance cannot be ignored. Therefore, it is necessary to identify resistance systems, including efflux pumps.

In the tracking section of efflux pumps encoding genes, $80 \%$ (48 samples) of 60 Acinetobacter baumanii isolates had adeA gene and $81.66 \%$ (49 samples) isolates had adeS gene, which compared to other studies conducted in Iran, Japanese race et al. [27] (adeA 100\%), Beheshti et al. [31] (for all gene pumps AdeABC 100\%) and Ardabili et al. [32] (adeS 100\%) were lower.

Compared to studies conducted abroad by Jia et al. In China [12], $79.6 \%$ of the Acinetobacter baumanii isolates had the adeA gene and $80.6 \%$ had the adeS gene, also in the study conducted by Nemec et al. in France [29] $81.9 \%$ of the isolates had adeA and adeS genes that were similar to the results of our study but compared to the research by Kor et al. [33] that the frequency of adeA gene in isolates was $62.7 \%$ that was higher. The reason for the differences in different studies could be due to differences in the patterns of antibiotic use, the type of clinical sample, the number of samples studied, sampling method, environmental factors and the different geographical distribution of these genes.

\section{Conclusion}

A significant increase in antibiotic resistance is one of the main problems in the treatment of infections caused by Acinetobacter baumannii. Efflux pumps play a key role in the development of multiple resistance to antimicrobial drugs. It is important to evaluate the presence of efflux pump genes in preventing the spread of antibiotic resistance and to suggest an appropriate treatment model for patients infected with this bacterium. So the high prevalence of genes encoding efflux pumps in this bacterium is one of the important factors in the spread of antibiotic resistance between isolates in different geographical regions. Of course, the role of other factors and mechanisms involved in the development of Acinetobacter baumannii resistance should not be ignored. 


\section{Limitations}

Our limitation in this research project was the lack of sufficient funding. If there is more budget, it can be further developed and more methods can be added.

\author{
Abbreviations \\ PCR: Polymerase chain reaction; CLSI: Clinical and Laboratory Standards Insti- \\ tute; MDR: Multi drug resistance; ATCC: American type culture collection; RND: \\ Resistance-nodulation-division; IMP: The genes imipenemase; VIM: Verona \\ integron-encoded metallo-beta-lactamases.
}

\section{Acknowledgements}

This study is financially supported by Shahed University of Medical Sciences in 2018. The authors of current study would like to express their deep thanks to all laboratory staff of Shahed University of Medical Sciences.

\section{Authors' contributions}

BBT, MN, MK: design of study. BBT, MK, HA, FS: acquisition of data. BBT, MN, RM, SB: evaluation of data, preparation of the manuscript. MK, HA, FS, SB: assessment of data. All authors read and approved the final manuscript.

\section{Funding}

This manuscript is part of a MSc thesis. This research was supported by the budget of research projects of the Shahed University of medical sciences. Funding were used to purchase equipment and tools.

\section{Availability of data and materials}

All relevant data are included in the manuscript.

\section{Ethics approval and consent to participate}

This study was in accordance with the declaration of Helsinki. This study was approved by the Ethics Committee of Shahed University of Medical Sciences. The informed consent was obtained from all the participants, and informed consent obtained was written.

\section{Consent for publication}

Not applicable.

\section{Competing interests}

The authors declare that they have no competing interests.

\section{Author details}

${ }^{1}$ Department of Microbiology, Faculty of Medicine, Shahed University, Tehran, Iran. ${ }^{2}$ Molecular Biology Research Center, System Biology and Poisoning Institute, Baqiyatallah University of Medical Sciences, Tehran, Iran.

Received: 4 August 2020 Accepted: 11 November 2020

Published online: 19 November 2020

\section{References}

1. Munier A-L, Biard L, Legrand M, Rousseau C, Lafaurie M, Donay J-L, et al. Incidence, risk factors and outcome of multi-drug resistant Acinetobacter baumannii nosocomial infections during an outbreak in a burn unit. IJID. 2019;79:179-84

2. Johanson WG Jr, Pierce AK, Sanford JP, Thomas GD. Nosocomial respiratory infections with gram-negative bacilli: the significance of colonization of the respiratory tract. Ann Intern Med. 1972;77(5):701-6.

3. Garnacho-Montero J, Timsit J-F. Managing Acinetobacter baumannii infections. Curr Opin Infect Dis. 2019;32(1):69-76.

4. Kaviani R, Pouladi I, Niakan M, Mirnejad R. Molecular detection of Adefg efflux pump genes and their contribution to antibiotic resistance in Acinetobacter baumannii clinical isolates. Rep Biochem Mol Biol. 2020;8(4):413.

5. Almasaudi SB. Acinetobacter spp. as nosocomial pathogens: epidemiology and resistance features. Saudi J Biol Sci. 2018;25(3):586-96.
6. Khaledi M, Abadi MSS, Validi M, Zamanzad B, Vafapour R, Gholipour A. Phenotypic and genotypic detection of metallo- $\beta$-lactamases in A. baumanii isolates obtained from clinical samples in Shahrekord, southwest Iran. BMC Res Notes. 2019;12(1):1-6.

7. Fan L, Wang Z, Wang Q, Xiong Z, Xu Y, Li D, et al. Increasing rates of Acinetobacter baumannii infection and resistance in an oncology department. J Cancer Res Ther. 2018;14(1):68.

8. Lee C-R, Lee JH, Park M, Park KS, Bae IK, Kim YB, et al. Biology of Acinetobacter baumannii: pathogenesis, antibiotic resistance mechanisms, and prospective treatment options. Front Cell Infect Microbiol. 2017;7:55.

9. Lin M-F, Lin Y-Y, Tu C-C, Lan C-Y. Distribution of different efflux pump genes in clinical isolates of multidrug-resistant Acinetobacter baumannii and their correlation with antimicrobial resistance. J Microbiol Immunol Infect. 2017;50(2):224-31.

10. Shahcheraghi F, Abbasalipour M, Feizabadi M, Ebrahimipour G, Akbari N. Isolation and genetic characterization of metallo- $\beta$-lactamase and carbapenamase producing strains of Acinetobacter baumannii from patients at Tehran hospitals. IJM. 2011:3(2):68.

11. Ghajavand H, Esfahani BN, Havaei SA, Moghim S, Fazeli H. Molecular identification of Acinetobacter baumannii isolated from intensive care units and their antimicrobial resistance patterns. Adv Biomed Res. 2015;4:110.

12. Jia W, Li C, Zhang H, Li G, Liu X, Wei J. Prevalence of genes of OXA-23 carbapenemase and AdeABC efflux pump associated with multidrug resistance of Acinetobacter baumannii isolates in the ICU of a comprehensive hospital of northwestern China. Int J Environ Res Public Health. 2015;12(8):10079-92

13. Turton JF, Woodford N, Glover J, Yarde S, Kaufmann ME, Pitt TL. Identification of Acinetobacter baumannii by detection of the blaOXA 51-like carbapenemase gene intrinsic to this species. J Clin Microbiol. 2006;44(8):2974-6.

14. Clinical, Laboratory. Standards Institute. Performance Standards for Antimicrobial Susceptibility Testing. M100-S27. 2017.

15. Sun J-R, Perng C-L, Chan M-C, Morita Y, Lin J-C, Su C-M, et al. A truncated AdeS kinase protein generated by IS Aba1 insertion correlates with tigecycline resistance in Acinetobacter baumannii. PLOS ONE. 2012;7(11):e49534.

16. Lopes B, Amyes S. Insertion sequence disruption of adeR and ciprofloxacin resistance caused by efflux pumps and gyrA and parC mutations in Acinetobacter baumannii. Int J Antimicrob Agents. 2013;41(2):117-21.

17. Thummeepak R, Kongthai P, Leungtongkam U, Sitthisak S. Distribution of virulence genes involved in biofilm formation in multi-drug resistant Acinetobacter baumannii clinical isolates. Int Microbiol. 2016:19(2):121-9.

18. Farsiani H, Mosavat A, Soleimanpour S, Nasab MN, Salimizand H, Jamehdar SA, et al. Limited genetic diversity and extensive antimicrobial resistance in clinical isolates of Acinetobacter baumannii in north-east Iran. J Med Microbiol. 2015;64(7):767-73.

19. Rynga D, Shariff M, Deb M. Phenotypic and molecular characterization of clinical isolates of Acinetobacter baumannii isolated from Delhi, India. Ann Clin Microbiol Antimicrob. 2015;14(1):40.

20. Noori M, Mohsenzadeh B, Bahramian A, Shahi F, Mirzaei H, Khoshnood $\mathrm{S}$. Characterization and frequency of antibiotic resistance related to membrane porin and efflux pump genes among Acinetobacter baumannii strains obtained from burn patients in Tehran, Iran. J Acute Dis. 2019;8(2):63.

21. Rahbarnia L, Farajnia S, Khaneshi H, Farajnia H, Naghili B, Tanomand A. Detection of blaOXA-23 and blaNDM-1 carbapenemase among clinical isolates of A. baumannii in Tabriz, north-west of Iran. Gene Rep. 2020;18:100555.

22. Abdar MH, Taheri-Kalani M, Taheri K, Emadi B, Hasanzadeh A, Sedighi A, et al. Prevalence of extended-spectrum beta-lactamase genes in Acinetobacter baumannii strains isolated from nosocomial infections in Tehran, Iran. GMS Hyg Infect Control. 2019;14:Doc02. https://doi.org/10.3205/ dgkh000318.

23. Fallah F, Noori M, Hashemi A, Goudarzi H, Karimi A, Erfanimanesh $\mathrm{S}$, et al. Prevalence of blaNDM, blaPER, blaVEB, blaIMP, and blaVIM genes among Acinetobacter baumannii isolated from two hospitals of Tehran, Iran. Scientifica. 2014;2014:245162.

24. Al-Agamy MH, Khalaf NG, Tawfick MM, Shibl AM, El Kholy A. Molecular characterization of carbapenem-insensitive Acinetobacter baumannii in Egypt. Int J Infect Dis. 2014;22:49-54. 
25. Angoti G, Bandehpour M, Goudarzi H, Hajizadeh M, Zarringhalam Moghaddam M, Kouchaki A. Detection of efflux pump genes (adeA, adeB, adeC and abeM) in Acinetobacter baumannii isolated from hospitalize patients, North-west of Iran. IEM. 2016;2(4):8-11.

26. Boral B, Unaldi Ö, Ergin A, Durmaz R, Eser ÖK. A prospective multicenter study on the evaluation of antimicrobial resistance and molecular epidemiology of multidrug-resistant Acinetobacter baumannii infections in intensive care units with clinical and environmental features. Ann Clin Microbiol Antimicrob. 2019;18(1):19.

27. Ranjbar R, Farahani A. Study of genetic diversity, biofilm formation, and detection of Carbapenemase, MBL, ESBL, and tetracycline resistance genes in multidrug-resistant Acinetobacter baumannii isolated from burn wound infections in Iran. Antimicrob Resist Infect Control. 2019;8(1):172.

28. Sarhaddi N, Soleimanpour S, Farsiani H, Mosavat A, Dolatabadi S, Salimizand $\mathrm{H}$, et al. Elevated prevalence of multidrug-resistant Acinetobacter baumannii with extensive genetic diversity in the largest burn centre of northeast Iran. JGAR. 2017;8:60-6.

29. Nemec A, Maixnerová M, van der Reijden TJ, Van den Broek PJ, Dijkshoorn L. Relationship between the AdeABC efflux system gene content, netilmicin susceptibility and multidrug resistance in a genotypically diverse collection of Acinetobacter baumannii strains. J Antimicrob Chemother. 2007;60(3):483-9.
30. Cortez-Cordova J, Kumar A. Activity of the efflux pump inhibitor phenylalanine-arginine $\beta$-naphthylamide against the AdeFGH pump of Acinetobacter baumannii. Int J Antimicrob Agents. 2011;37(5):420-4.

31. Beheshti M, Talebi M, Ardebili A, Bahador A, Lari AR. Detection of AdeABC efflux pump genes in tetracycline-resistant Acinetobacter baumannii isolates from burn and ventilator-associated pneumonia patients. J Pharm Bioallied Sci. 2014;6(4):229.

32. Ardebili A, Talebi M, Azimi L, Lari AR. Effect of efflux pump inhibitor carbonyl cyanide 3-chlorophenylhydrazone on the minimum inhibitory concentration of ciprofloxacin in Acinetobacter baumannii clinical isolates. Jundishapur J Microbiol. 2014;7(1):e8691.

33. Kor S-B, Beatrice-Sing-Yieng Tou C-K, Chieng L, Michele-Sook-Yuin Hiew $\mathrm{C}-\mathrm{H}$. Distribution of the multidrug efflux pump genes adeA, adel, adeJ, adeY and integrons in clinical isolates of Acinetobacter baumannii from Malaysian hospitals. Biomed. Res. 2014;25(2):1-6.

\section{Publisher's Note}

Springer Nature remains neutral with regard to jurisdictional claims in published maps and institutional affiliations.
Ready to submit your research? Choose BMC and benefit from:

- fast, convenient online submission

- thorough peer review by experienced researchers in your field

- rapid publication on acceptance

- support for research data, including large and complex data types

- gold Open Access which fosters wider collaboration and increased citations

- maximum visibility for your research: over $100 \mathrm{M}$ website views per year

At BMC, research is always in progress.

Learn more biomedcentral.com/submissions 